\title{
Earth Mover's Prototypes: \\ A Convex Learning Approach for \\ Discovering Activity Patterns in \\ Dynamic Scenes
}

Gloria Zen ${ }^{1}$, Elisa Ricci ${ }^{2,3}$

\author{
${ }^{1} \mathrm{DISI}$, University of Trento, Trento, Italy. \\ 2 TEV, Fondazione Bruno Kessler (FBK), Trento, Italy. \\ ${ }^{3}$ DIEI, University of Perugia, Italy.
}

International Conference Computer Vision and Pattern Recognition (CVPR), 2011 


\section{Mining behaviors in complex scenes}

\section{Goals:}

i) to mine patterns of recurrent activities (e.g. vertical/horizontal traffic flows)
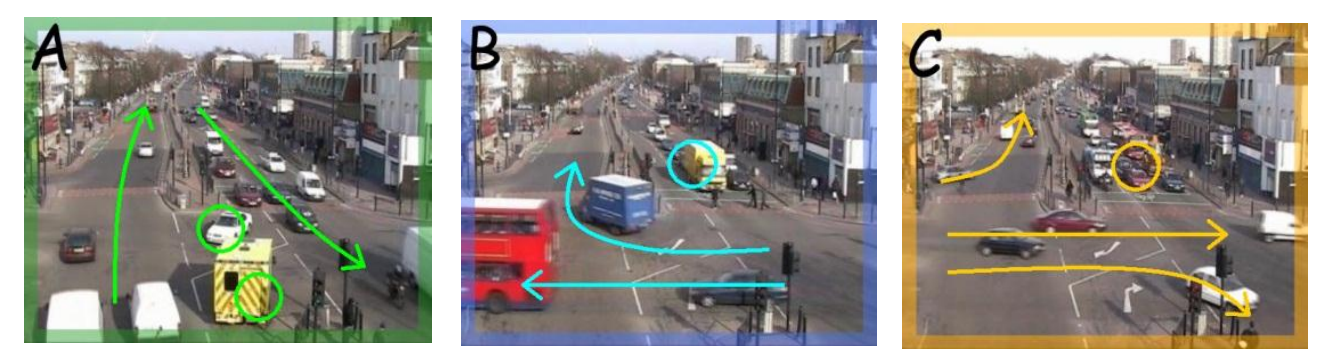

ii) to detect anomalies

(e.g. jaywalker, accident, unusual patterns)
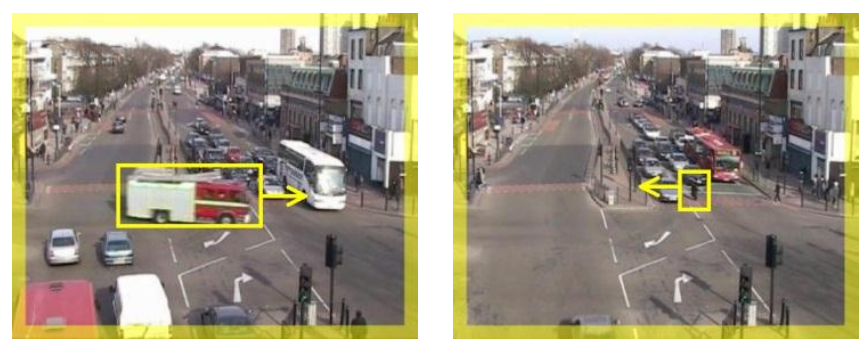

video:

junction.avi

Object-centric methods

- occlusions (broken trajectories)

Non-object-centric methods

- several targets (curse of dimensionality)

Not reliable! 


\section{Non-object centric methods in a nutshell}

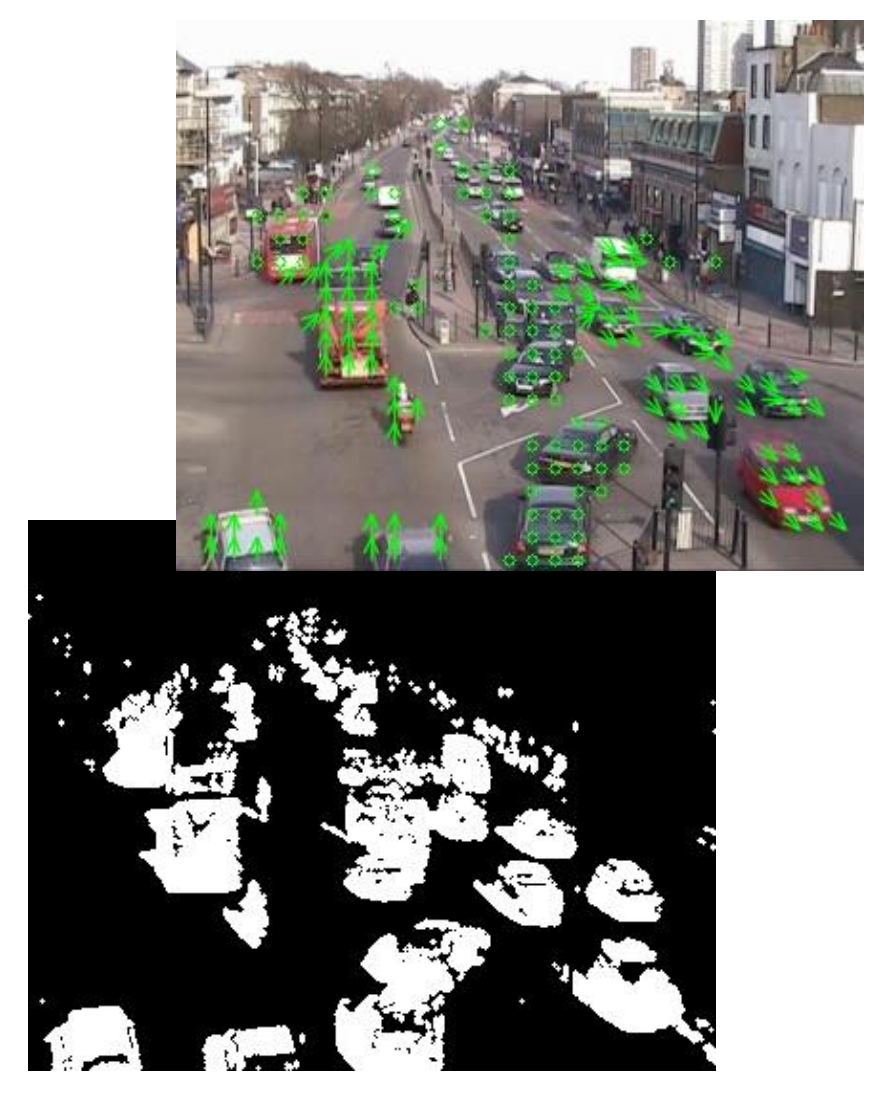

- Low level cues (optical flow, foreground) are extracted and quantized (position and motion) to generate visual words.

- Short video clips are represented as visual documents.

- Salient activities (topics) of the scenes are extracted based on Probabilistic Topic Models (PTMs) [Kuettel10, Varadarajan10, Hospedales11].

Dependencies between atomic activities (words) are not considered! 


\section{Our approach}
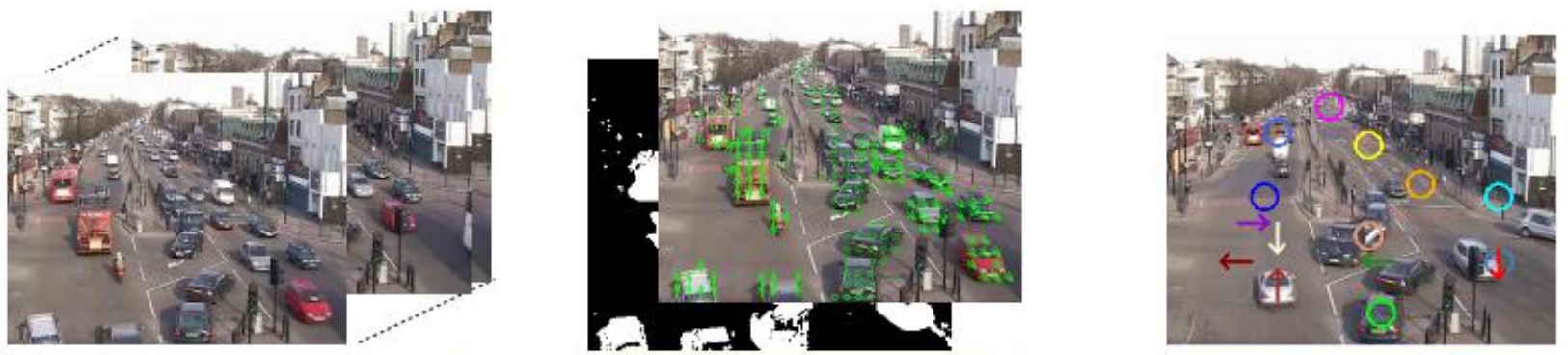

\section{Step A}

detect atomic activities (codebook generation)

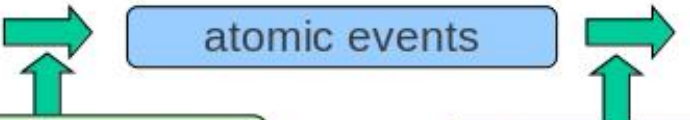

atomic activities

low level features

k-medoids

a

Step B

(main contribution) extract patterns of typical activities
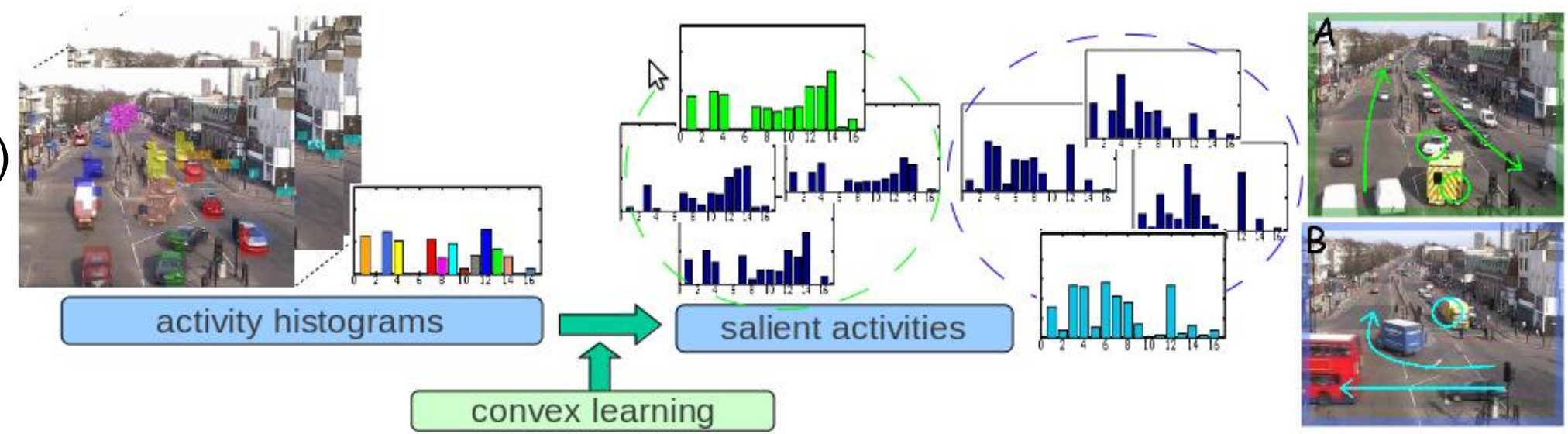


\section{Main contributions}

- The task of extracting typical activities is formulated as a simple linear programming (LP) problem.

- The similarity between atomic activities is considered by adopting a variation of the Earth Mover Distance (EMD) as distance measure between histograms.

- Anomalous patterns are detected by comparing salient activities extracted at multiple scales. 


\section{1/3: Convex prototype learning}

Given a set of histograms:

$$
\mathcal{H}=\left\{\boldsymbol{h}_{1}, \ldots, \boldsymbol{h}_{N}\right\}, \boldsymbol{h}_{i} \in \mathbb{R}^{D}
$$

We aim to learn $N$ representative prototypes:

$$
\mathcal{P}=\left\{\boldsymbol{p}_{1}, \ldots, \boldsymbol{p}_{N}\right\}, \boldsymbol{p}_{i} \in \mathbb{R}^{D}
$$

This task is formalized as a convex optimization problem:

$$
\min _{\boldsymbol{p}_{i} \in \boldsymbol{\Omega}} \underbrace{\sum_{i=1}^{N} \mathcal{L}\left(\boldsymbol{h}_{i}, \boldsymbol{p}_{i}\right)}_{\text {LoSS }}+\lambda \underbrace{\sum_{i \neq j} \eta_{i j} \mathcal{J}\left(\boldsymbol{p}_{i}, \boldsymbol{p}_{j}\right)}_{\text {Regularization }}
$$

Loss: similarity between prototype $\boldsymbol{p}_{i}$ and associated histogram $\boldsymbol{h}_{i}$

Regularization: smoothness among neighboring prototypes ( $\boldsymbol{p}_{i}$ is fused into $\boldsymbol{p}_{j}$ ) $\eta_{i j}=\{0,1\}$ indicates prototype's neighborhood

Temporal segmentation: $\eta_{i j}$ based on a temporal distance criterion Clustering: $\eta_{i j}$ based on the distance between histograms' values 


\section{1/3: Convex prototype learning}

Given the objective function:

$$
\min _{\boldsymbol{p}_{i} \in \boldsymbol{\Omega}} \sum_{i=1}^{N} \mathcal{L}\left(\boldsymbol{h}_{i}, \boldsymbol{p}_{i}\right)+\lambda \sum_{i \neq j} \eta_{i j} \max _{q=1 \ldots D}\left|p_{i}^{q}-p_{j}^{q}\right|
$$

What happens in practice:

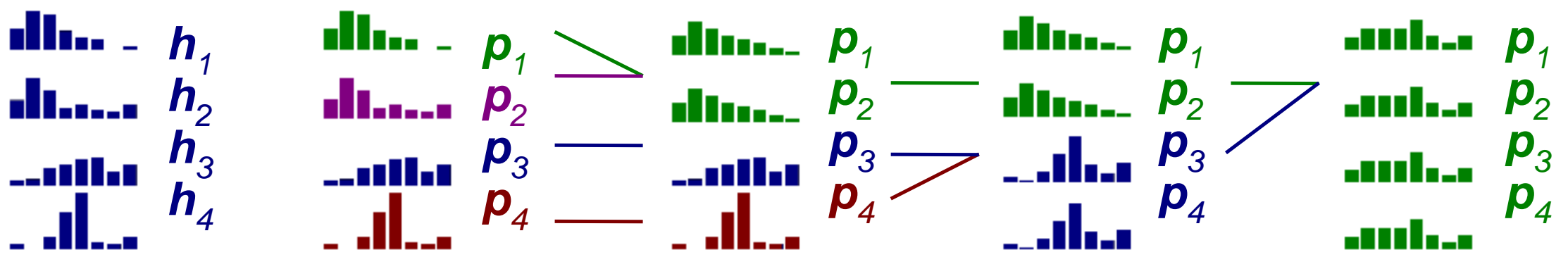

$\lambda=0 \quad \lambda$ controls the number of
different prototypes!

Resulting dendrogram:

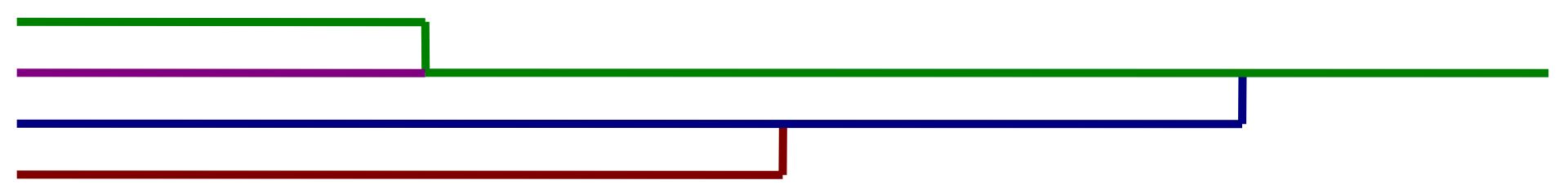




\section{2/3: Multi-scale analysis}

- Comparing clustering results at multiple scales we can detect unusual behaviors corresponding to atypical histograms.

- A high Multiscale Anomaly Score (MAS) is assigned to small clusters which persist (do not fuse) along the multi-scale analysis.

- Detection of a jaywalker
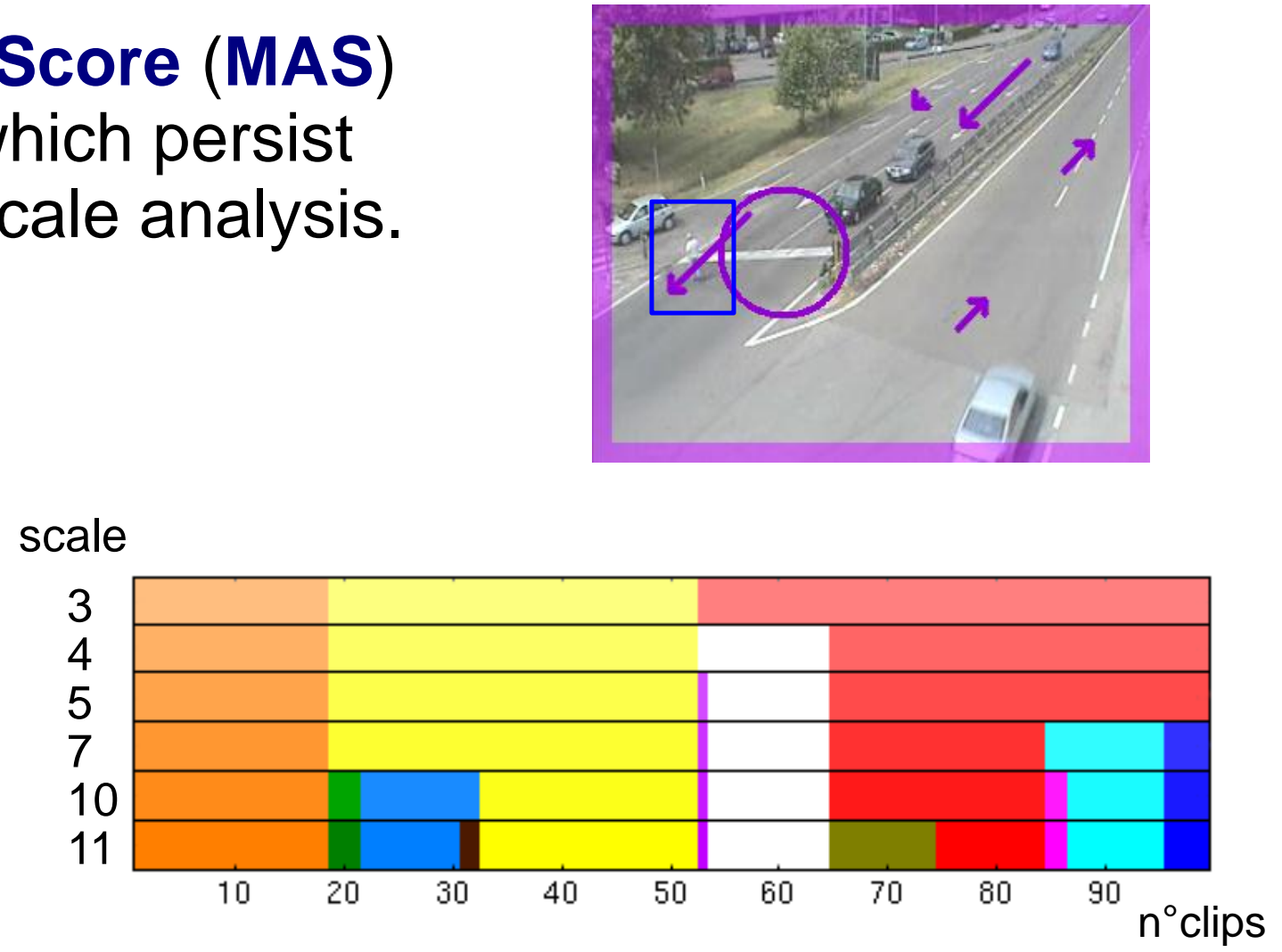


\section{3/3: Correlation among activities}

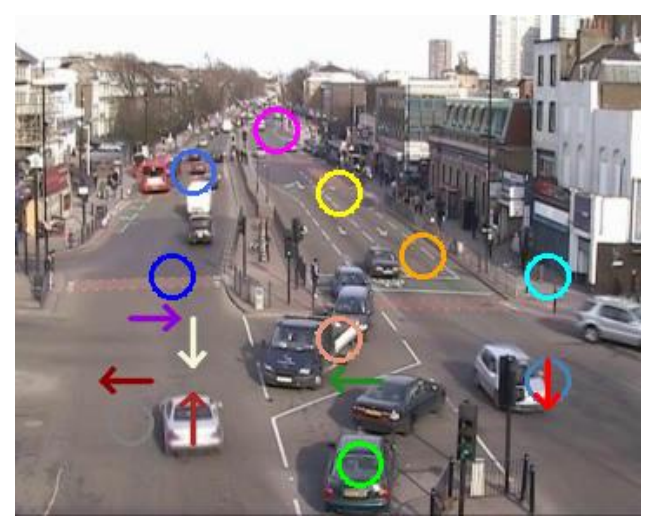

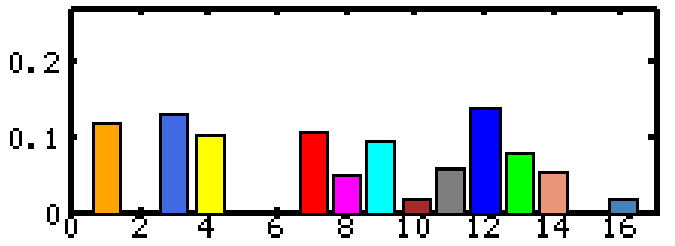

Histogram of activities for corresponding clip
We want to consider similarity among activities when comparing clip histograms

Atomic activities

A cross-bin (Earth Mover Distance) instead of a simple bin-to-bin distance is adopted:

$E M D(\boldsymbol{h}, \boldsymbol{k})=\min _{f_{q t} \geq 0} \sum_{q, t=1}^{D} d_{q t} f_{q t} \quad$ s.t. $\sum_{q=1}^{D} f_{q t}=h^{t}, \sum_{t=1}^{D} f_{q t}=k^{q}$

$f_{q t}$ : amount of flow we want to transfer from bin $q$ to $t$.

$d_{q t}:$ ground distance, encodes similarity among activities.

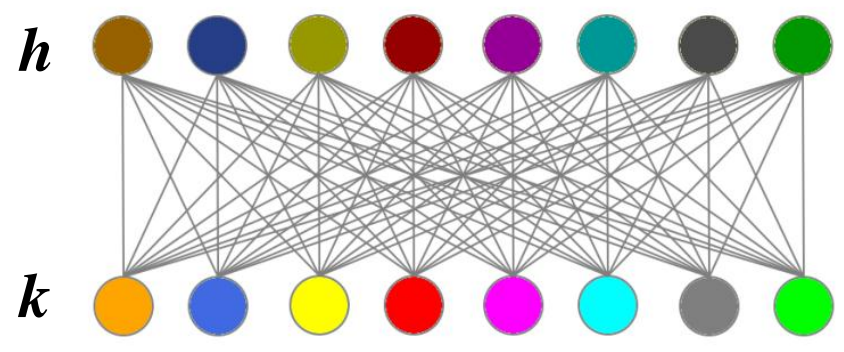




\section{3/3: Earth Mover's Prototypes}

1) We adopt the EMD in the Loss function:

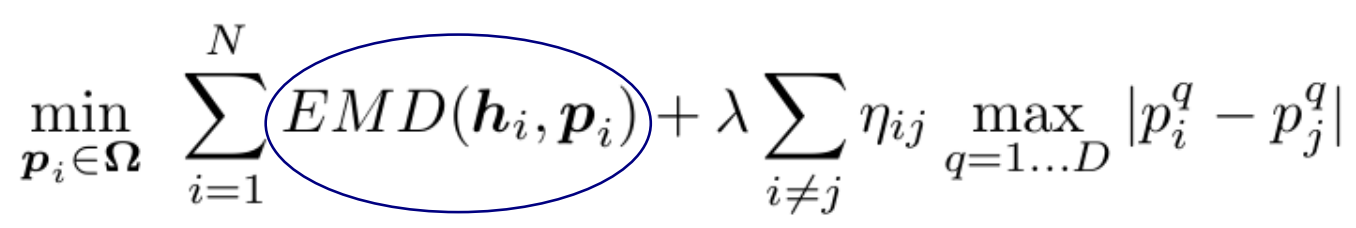

Complexity: $O\left(D^{2}\right)$. This is computationally expensive...

2) An efficient variation of EMD (EMD- $\left.L_{1}\right)$ is adopted [Ling06], with $L_{1}$ distance over bins as ground distance:

$$
d_{q t}=|q-t|
$$

$E M D$

$$
\longrightarrow E M D-L_{1}
$$

Complexity: reduced from $O\left(D^{2}\right)$ to $O(D)$ !

Sorting: similar activities must correspond to neighboring bins in the histogram!

3) A bin-to-bin distance $\left(L_{1}\right)$ is also considered for performance evaluation. Complexity: $O(D)$ 


\section{3/3 Earth Mover's Prototypes}

The overall optimization problem is a parametric LP:

$$
\begin{array}{cl}
\min _{\boldsymbol{p}_{i}, f_{q t}^{i} \geq 0} & \sum_{i=1}^{N} \sum_{q, t=1}^{D} d_{q t} f_{q t}^{i}+\lambda \sum_{i \neq j} \eta_{i j} \max _{q=1 \ldots D}\left|p_{i}^{q}-p_{j}^{q}\right| \\
\text { s.t. } & \sum_{q=1}^{D} f_{q t}^{i}=h_{t}^{i}, \quad \sum_{t=1}^{D} f_{q t}^{i}=p_{q}^{i}
\end{array}
$$

A variant of the revised simplex method can be used to compute the entire regularization path:

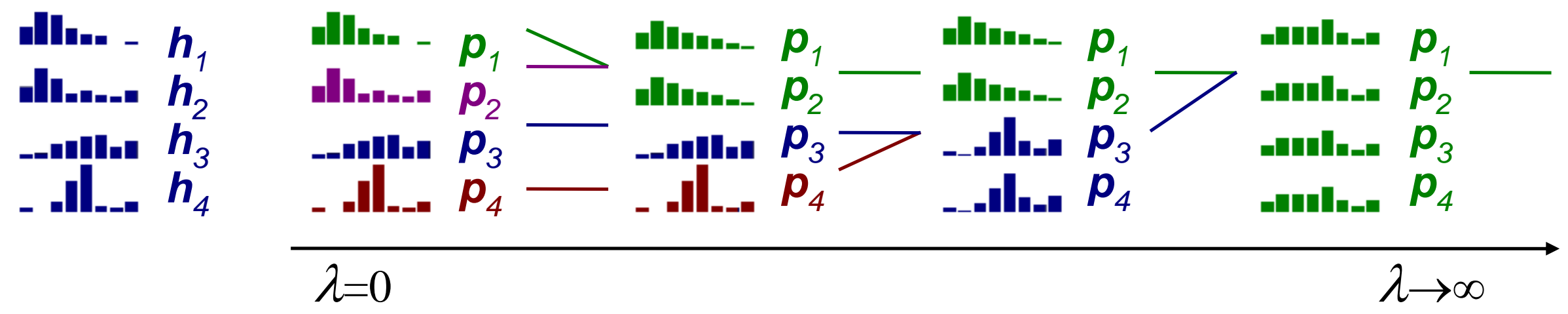




\section{Results: datasets}

We tested our method on 4 datasets ( 3 of them publicly available):

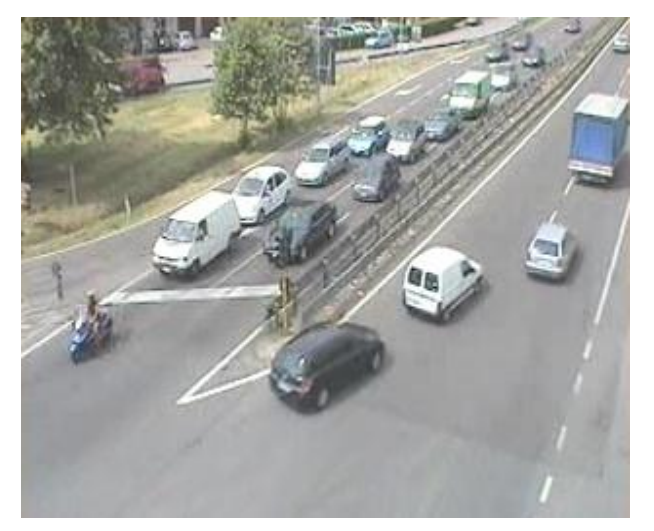

\section{Traffic}

public no

$n^{\circ}$ frames fps 6000 12 frame size $276 \times 336$

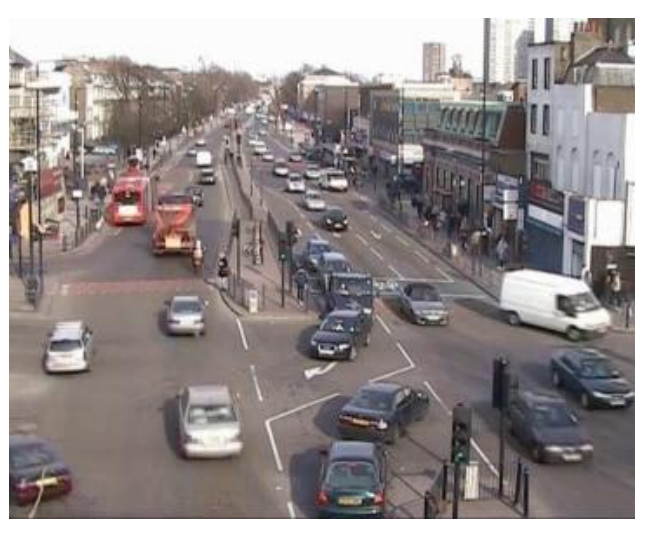

\begin{tabular}{lr}
\hline Junction & \\
public & yes \\
$n^{\circ}$ frames & 90000 \\
fps & 25 \\
frame size & $288 \times 360$
\end{tabular}

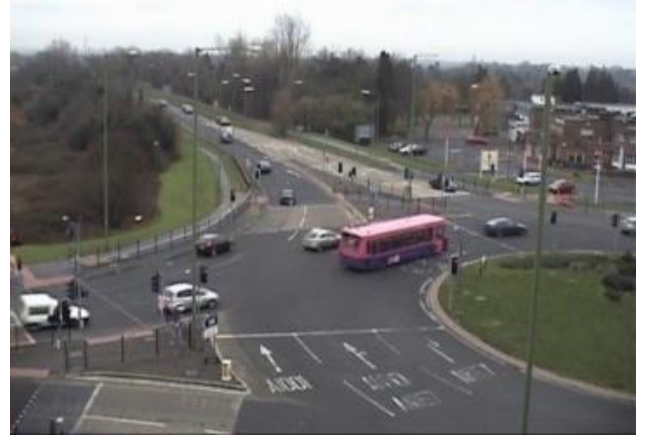

\begin{tabular}{lr}
\hline \multicolumn{2}{|l}{ Roundabout } \\
public & yes \\
$\mathrm{n}^{\circ}$ frames & 93500 \\
fps & 25 \\
frame size & $288 \times 360$
\end{tabular}

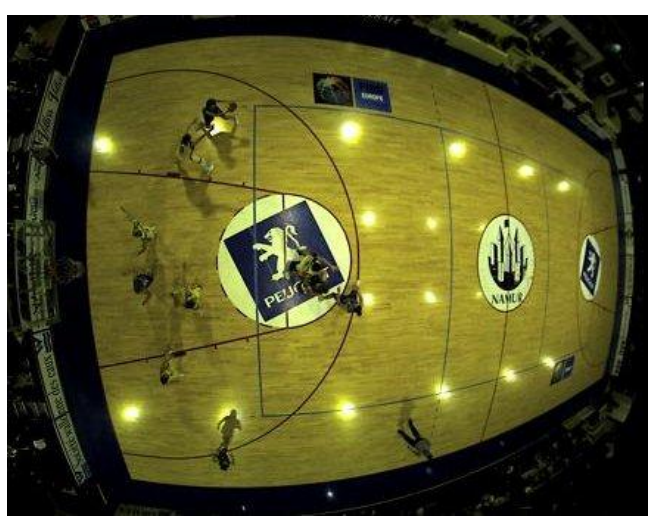

Basket - APIDIS ${ }^{2}$

public yes

$n^{\circ}$ frames 6000 fps 23 frame size $\quad 320 \times 368$

${ }^{1}$ QMUL dataset, available on www.eecs.qmul.ac.uk/ jianlil

${ }^{2}$ APIDIS dataset, available on www. apidis. org/Dataset/ 


\section{Results: multiscale analysis}

\begin{tabular}{|lr|}
\hline Junction dataset \\
\hline $\mathrm{n}^{\circ}$ clips & 120 \\
cliplen & 375 \\
$\mathrm{n}^{\circ}$ activities & 16 \\
$\begin{array}{l}\text { Tot video } \\
\text { duration }\end{array}$ & $30 \mathrm{~min}$ \\
\hline
\end{tabular}

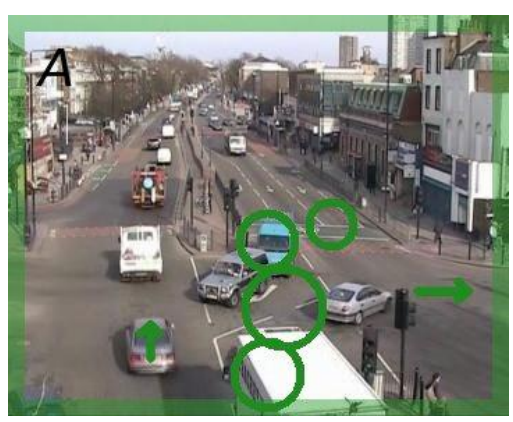

\section{Vertical}

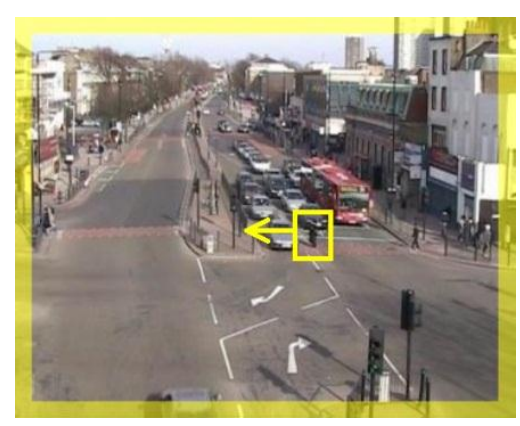

u1: Jaywalker

3 clusters

5 clusters \begin{tabular}{|l|l|l|l|l|l|l|l|l|}
\hline \\
\hline
\end{tabular}

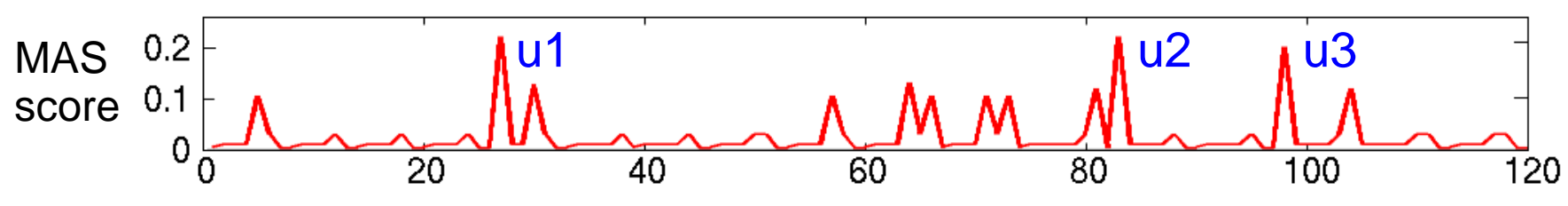

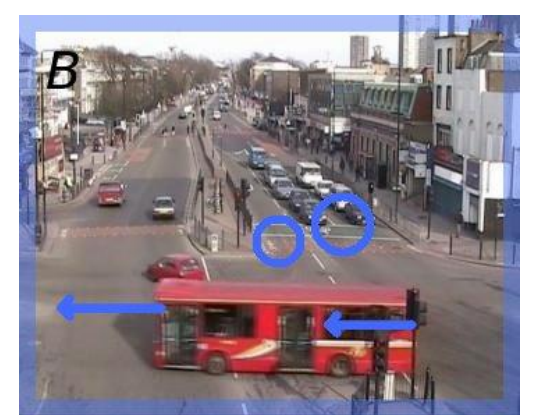

Horizontal $\longleftarrow$

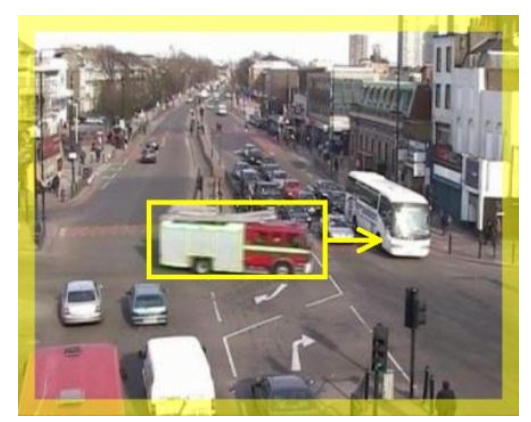

u2: Fire engine

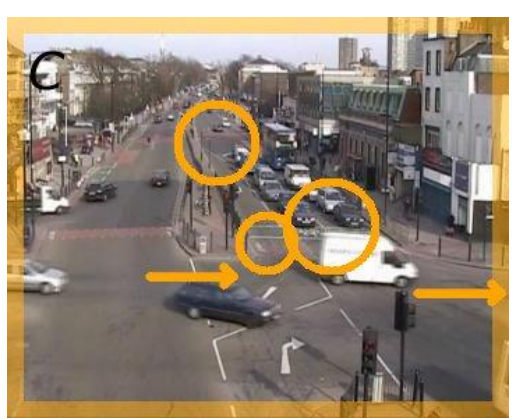

Horizontal $\rightarrow$

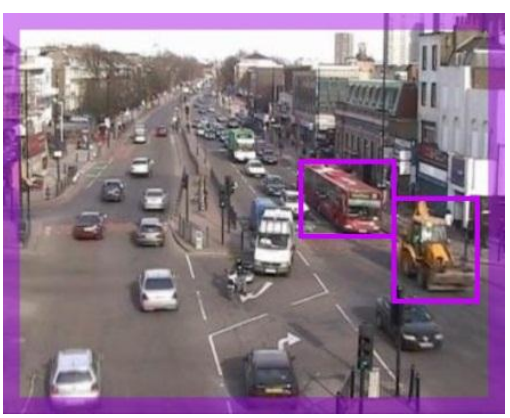

u3: Heavy traffic
- Three main traffic flows

- Unusual events

video: junction.avi 


\section{Results: clustering}

- Salient activities discovered

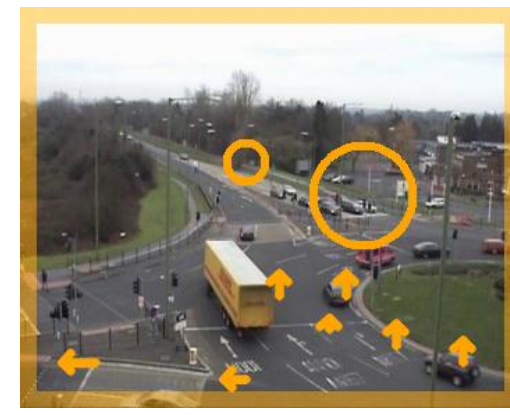

Vertical flow

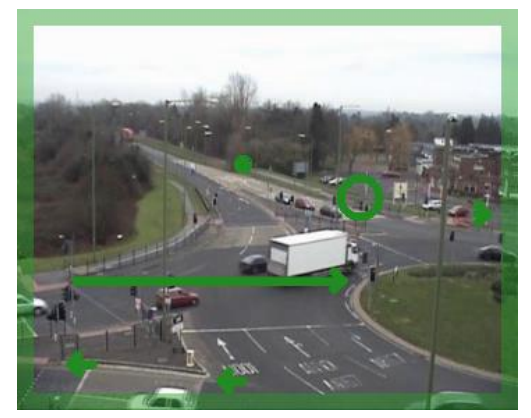

Horizontal flow video:

roundabout.avi
Roundabout dataset

$n^{\circ}$ clips

cliplen 300

$\mathrm{n}^{\circ}$ activities 16

Tot video duration

- Accuracy (ground truth ${ }^{1}$ )

\begin{tabular}{|l|ccccc|}
\hline & EMD-L $_{1}$ & $\mathrm{~L}_{1}$ & $\begin{array}{c}\text { EMD-L } \\
\text { random }\end{array}$ & $\begin{array}{c}\text { Standard pLSA } \\
\text { [Li08bmvc] }\end{array}$ & $\begin{array}{c}\text { Hierarchical pLSA } \\
\text { [Li08bmvc] }\end{array}$ \\
\hline Junction & $\mathbf{9 2 . 3 6}$ & 89.74 & 86.7 & 89.74 & 76.92 \\
Roundabout & $\mathbf{8 6 . 4 0}$ & $\mathbf{8 6 . 4 0}$ & 72.3 & 84.46 & 72.30 \\
\hline
\end{tabular}

${ }^{1}$ [Li08bmvc] J. Li, S. Gong, and T. Xiang. Global behaviour inference using probabilistic latent semantic analysis. BMVC, 2008 


\section{Results: basket dataset}

\section{- Five salient activities discovered}

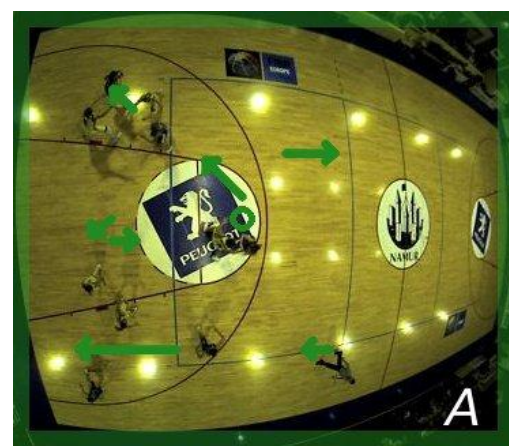

Blue team on attack

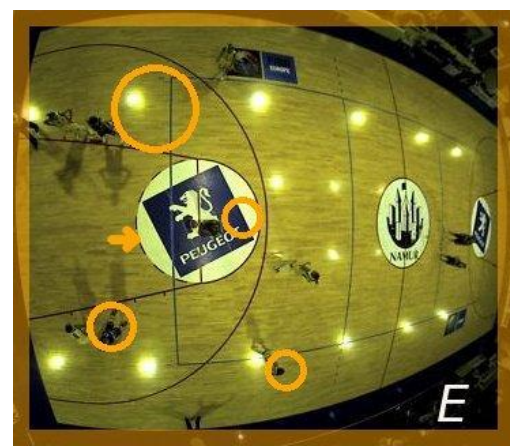

Blue team in a free-throw

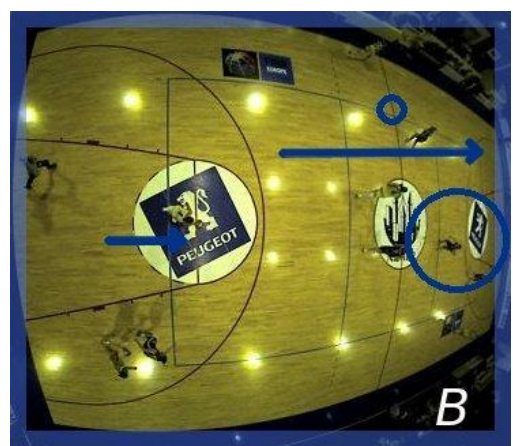

Towards blue team's court

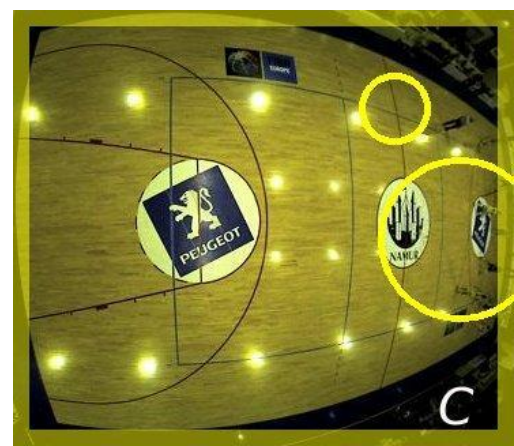

Yellow team on attack

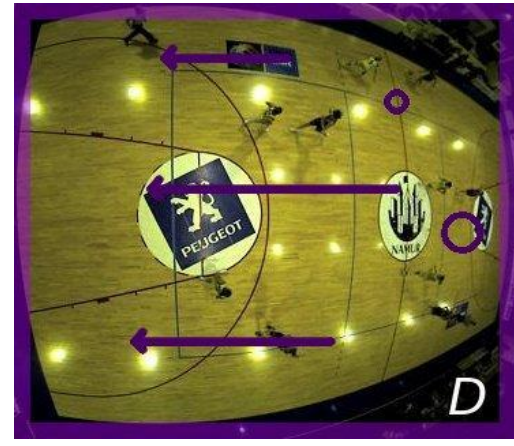

Towards yellow team's court

\section{- Accuracy}

Ground truth EMD-L1

L1

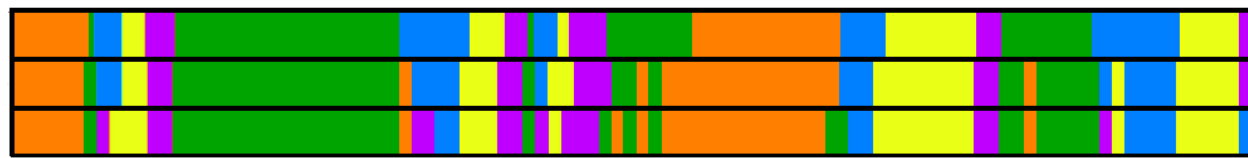

\begin{tabular}{|c|cccc|}
\hline $\mathrm{n}^{\circ}$ clusters & EMD-L $_{1}$ & $\mathrm{~L}_{1}$ & $\mathrm{pLSA}$ & $\mathrm{pLSA}-$ bin \\
\hline 5 & $\mathbf{9 0 . 8 4}$ & 75.17 & 83.5 & 77.5 \\
2 & $\mathbf{9 8 . 4 2}$ & $\mathbf{9 8 . 4 2}$ & 94.15 & 92.25 \\
\hline
\end{tabular}

video: basket.avi

\begin{tabular}{lr} 
Basket dataset & \\
$\mathrm{n}^{\circ}$ clips & 100 \\
cliplen & 60 \\
$\mathrm{n}^{\circ}$ activities & 16 \\
$\begin{array}{l}\text { Tot video } \\
\text { duration }\end{array}$ & $5 \mathrm{~min}$ \\
\hline
\end{tabular}




\section{Conclusions}

- Our approach has shown to be effective for multiscale analysis of complex video scenes. It relies on a convex optimization problem.

- Up to our knowledge this is the first work which considers the similarity between the atomic activities.

-A variant of the EMD allows to embed this information at a feasible cost, while the sorting of atomic activities in the histogram becomes crucial for good performance.

- Future work will focus on improving scalability (ad hoc solver needed) and learning of temporal rules.

Data and code will be available on: www.disi.unitn.it/ zen 


\section{References}

[Hospedales11] T. Hospedales, J. Li, S. Gong, T. Xiang. Identifying Rare and Subtle Behaviours: A Weakly Supervised Joint Topic Model. IEEE Trans. on PAMI, 2011

[Kuettel10] D. Kuettel, M. D. Breitenstein, L. V. Gool, and V. Ferrari. What's going on? Discovering spatio-temporal dependencies in dynamic scenes. CVPR, 2010.

[Varad10] J. Varadarajan, R. Emonet, and J.-M. Odobez. Probabilistic latent sequential motifs: Discovering temporal activity patterns in video scenes. BMVC, 2010

[Hospedales09] T. Hospedales, S. Gong, and T. Xiang. A markov clustering topic model for mining behaviour in video. ICCV, 2009.

[Yang09] Y. Yang, J. Liu, and M. Shah. Video scene understanding using multi-scale analysis. ICCV, 2009.

[Li08] J. Li, S. Gong, and T. Xiang. Scene segmentation for behaviour correlation. ECCV, 2008.

[Li08b] J. Li, S. Gong, and T. Xiang. Global behaviour inference using probabilistic latent semantic analysis. BMVC, 2008.

[Ling06] H. Ling and K. Okada. An efficient Earth Mover's Distance algorithm for robust histogram comparison. IEEE Trans. on PAMI, 2006 


\section{Results: temporal segmentation}

- Traffic dataset

- Salient activities
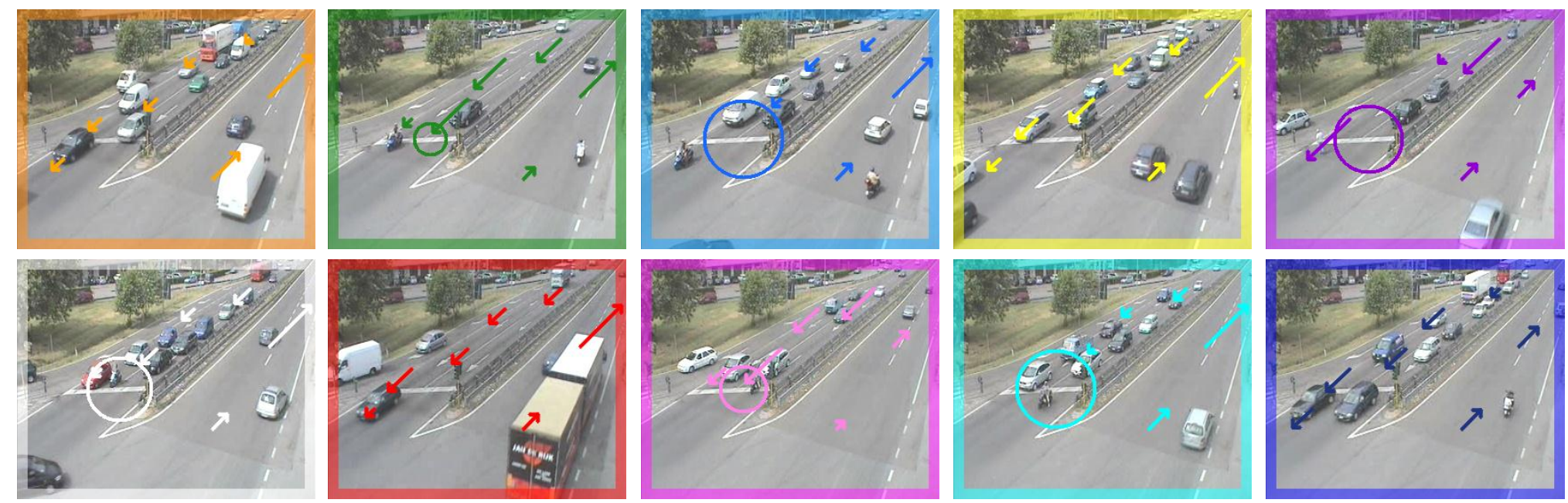

video: traffic.avi

\section{- Accuracy}

Ground truth(12)

Ground truth(7)

EMD-L1

L1

Fused Lasso

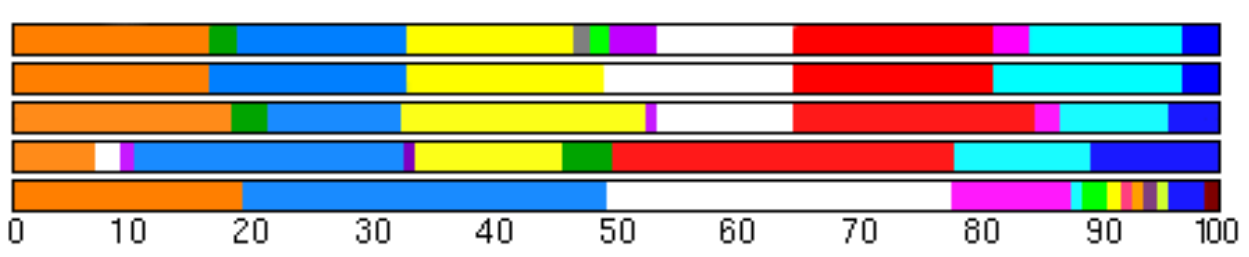

\begin{tabular}{|ccc|}
\hline EMD-L & $\mathrm{L}_{1}$ & Fused Lasso \\
\hline 82.4 & 72.5 & 68.7 \\
\hline
\end{tabular}

